\title{
Kepler equation for inspiralling compact binaries
}

\author{
Zoltán Keresztes, Balázs Mikóczi, and László Á. Gergely \\ Departments of Theoretical and Experimental Physics, University of Szeged, Szeged 6720, Hungary
}

(Received 20 October 2005; published 28 November 2005)

\begin{abstract}
Compact binaries consisting of neutron stars/black holes on eccentric orbit undergo a perturbed Keplerian motion. The perturbations are either of relativistic origin or are related to the spin, mass quadrupole, and magnetic dipole moments of the binary components. The post-Newtonian motion of such systems decouples into radial and angular parts. We present here for the first time the radial motion of such a binary encoded in a generalized Kepler equation, with the inclusion of all above-mentioned contributions, up to linear order in the perturbations. Together with suitably introduced parametrizations, the radial motion is solved completely.
\end{abstract}

DOI: 10.1103/PhysRevD.72.104022

PACS numbers: 04.30.Db, 04.25.Nx

\section{INTRODUCTION}

The worldwide effort to capture gravitational wave signals emitted by astrophysical sources is under way. A network of interferometric gravitational wave detectors [1-4] is either already operational, or close to completion. Compact binaries consisting on neutron stars/black holes are among the best candidates to emit gravitational radiation in the bandwidth of these detectors. Upper limits on the gravitational radiation emitted by such binaries were already found $[5,6]$ from the S2 scientific run of LIGO.

The evolution of such a binary system can be divided into three phases: inspiral, merger and ringdown. The merger phase can be understood only by numeric simulations. Even in the last part of the inspiral a numerical treatment seems adequate due to the intermediate binary black hole (IBBH) problem [7], studied also in [8]. Earlier, in the inspiral phase a post-Newtonian (PN) description of high accuracy provides satisfactory results. The PN corrections of relativistic nature are known to 3PN orders [9]. However there are other contributions to be taken into account, related to various physical characteristics of the binary components as well.

For compact binaries, there is the spin-orbit (SO) interaction appearing at $1.5 \mathrm{PN}$ orders. At this accuracy both the spin vectors $\mathbf{S}_{\mathbf{i}}$ and the orbital angular momentum $\mathbf{L}$ undergo a precessional motion about the total angular momentum $\mathbf{J}$ [10]. This is a novel feature in the postNewtonian evolution of the system. Such an effect was recently claimed [11] to be observable for the J07373039A/B double pulsar [12], [13].

The precessional motion of the spin(s) is called simple precession, whenever the two masses are equal, or one of the spins can be neglected, say $\mathbf{S}_{\mathbf{2}}=0$ [14]. These two cases were studied in [15], where among other results, the Kepler equation was derived up to 3PN orders with the inclusion of the SO contributions. As a related result, the evolution of the relativistic periastron advance parameter was recently computed [16]. The tilt angle of the spin with respect to $\mathbf{L}$ was estimated to be smaller than $\simeq 60^{\circ}$ from generic astrophysical considerations on the evolution of compact binaries [17].

The SO interaction gives corrections to the losses of energy and magnitude of angular momentum of the system occurring due to gravitational radiation. For eccentric orbits these were given by $[18,19]$. An other work relying on the use of the effective one-body approach [20], [21], has employed the SO contribution in the study of the inspiral to plunge phase of the coalescence [22].

Moreover, in the two cases of simple precession detection template families have been worked out both containing a set of phenomenological parameters [23] or physical parameters [24]. The latter would allow for determining the angle $\kappa_{1}$ and the magnitude of the single spin (in fact of $\chi=S_{1} / m_{1}^{2}$ ) from the study of gravitational radiation.

At $2 \mathrm{PN}$ another set of new effects related to various physical characteristics of the compact binary emerge. The losses of energy and magnitude of angular momentum of the system on eccentric orbit, due to gravitational radiation were derived in [25] for the spin-spin interaction, in [26] for the mass quadrupole-monopole interaction and in [27] for the interaction of magnetic dipoles.

Thus at $2 \mathrm{PN}$ physical quantities like the mass quadrupole and magnetic dipole moments, as well as angular variables characterizing the spins and moments appear in the formalism. Neither detection templates, nor methods to find out these new physical parameters have been worked out so far. We note that in principle, the observation of the evolution of gravitational wave frequency [28] allows to impose constraints on a combination of these parameters, but does not allow to predict their individual values. There is still much to do until a complete understanding of the complicated motion the system, occurring when all these interactions are taken into account, will be achieved. Our present work fills an important gap in the description of compact binaries with the enlisted physical characteristics.

In Sec. II we describe the post-Newtonian motion of such a binary system. The radial part of the motion decouples and defines a radial orbit. We give the generic expressions of the turning points of the radial motion which 
allows for the introduction of the semimajor axis $a_{r}$ and radial eccentricity $e_{r}$. We define the generalized true anomaly $\chi$ and eccentric anomaly $\xi$ parameters. Our generalized true anomaly parameter is different from the one employed in the Damour-Deruelle formalism and we establish their relation. In Sec. III we derive the main result of the paper, which is the generalized Kepler equation

$$
\begin{aligned}
n\left(t-t_{0}\right)= & \xi-e_{t} \sin \xi+f_{t} \sin \left[\chi+2\left(\psi_{0}-\bar{\psi}\right)\right] \\
& +\sum_{i=1}^{2} f_{t}^{i} \sin \left[\chi+2\left(\psi_{0}-\psi_{i}\right)\right] .
\end{aligned}
$$

The explicit expressions of the orbital elements $n, e_{t}, f_{t}$, and $f_{t}^{i}$ are given in Sec. IV, together with all contributions to $a_{r}$ and $e_{r}$. They contain the relativistic contributions (PN terms), together with the SO, SS, DD and QM terms, all to linear order. One of the consequences of taking into account the physical characteristics of the binary, like the spins, mass quadrupole and magnetic dipole moments is the emergence of novel angle variables in the Kepler equation (1). The angles $\psi_{i}$ are the azimuthal angles of the spins and $2 \bar{\psi}=\psi_{1}+\psi_{2}$. (For more details on the notations see [19].) The angle $\psi_{0}$ is the argument of the periastron (defined here as the angle subtended by the periastron and the intersection line of the planes perpendicular to the total and orbital angular momenta, respectively).

We emphasize that in the description of the SO interaction several spin-supplementary conditions (SSC) can be used. In order to simplify the formalism, in this paper we use the noncovariant SSC of Pryce [29] and Newton and Wigner [30]. In this SSC the Lagrangian is not acceleration dependent and the radial equation is simpler than in the covariant SSC [29] employed earlier in [19].

\section{GENERALIZED TRUE AND ECCENTRIC ANOMALY PARAMETRIZATIONS}

The linear contributions to the motion of the compact binary can be collected in the Lagrangian

$$
\mathcal{L}=\mathcal{L}_{N}+\mathcal{L}_{\mathrm{PN}}+\mathcal{L}_{\mathrm{SO}}+\mathcal{L}_{\mathrm{SS}}+\mathcal{L}_{\mathrm{QM}}+\mathcal{L}_{\mathrm{DD}},
$$

with the various contributions derived first in [31] $(\mathrm{PN})$, [32] (SS), [33] (QM) and [34] (DD):

$$
\begin{aligned}
& \mathcal{L}_{N}=\frac{\mu \mathbf{v}^{2}}{2}+\frac{G m \mu}{r}, \quad \mathcal{L}_{\mathrm{PN}}=\frac{1}{8 c^{2}}(1-3 \eta) \mu v^{4}+\frac{G m \mu}{2 r c^{2}}\left[(3+\eta) v^{2}+\eta \dot{r}^{2}-\frac{G m}{r}\right], \\
& \mathcal{L}_{\mathrm{SO}}=\frac{G \mu}{2 c^{2} r^{3}} \mathbf{v} \cdot[\mathbf{r} \times(4 \mathbf{S}+3 \boldsymbol{\sigma})], \quad \mathcal{L}_{\mathrm{SS}}=\frac{G}{c^{2} r^{3}}\left[\left(\mathbf{S}_{\mathbf{1}} \cdot \mathbf{S}_{\mathbf{2}}\right)-\frac{3}{r^{2}}\left(\mathbf{r} \cdot \mathbf{S}_{\mathbf{1}}\right)\left(\mathbf{r} \cdot \mathbf{S}_{\mathbf{2}}\right)\right], \\
& \mathcal{L}_{\mathrm{QM}}=\frac{G \mu m^{3}}{2 r^{5}} \sum_{i=1}^{2} p_{i}\left[3\left(\hat{\mathbf{S}}_{\mathbf{i}} \cdot \mathbf{r}\right)^{2}-r^{2}\right], \quad \mathcal{L}_{\mathrm{DD}}=\frac{1}{r^{3}}\left[3\left(\mathbf{n} \cdot \mathbf{d}_{\mathbf{1}}\right)\left(\mathbf{n} \cdot \mathbf{d}_{\mathbf{2}}\right)-\mathbf{d}_{\mathbf{1}} \cdot \mathbf{d}_{\mathbf{2}}\right] .
\end{aligned}
$$

Note that the SO part of the Lagrangian above was not given before and it is valid when the spin-supplementary condition (SSC) of Pryce [29] and Newton and Wigner [30] is chosen. We have verified that the SO part of the acceleration derived from $\mathcal{L}_{\text {SO }}$ agrees with the expression (A1b) of [35]. The magnitude and direction of the spins are denoted as $S_{i}$ and $\hat{\mathbf{S}}_{\mathbf{i}}$. The angle subtended by them is $\gamma=$ $\cos ^{-1}\left(\hat{\mathbf{S}}_{\mathbf{1}} \cdot \hat{\mathbf{S}}_{\mathbf{2}}\right)$. Here $\mathbf{S}=\mathbf{S}_{\mathbf{1}}+\mathbf{S}_{\mathbf{2}}$ and $\boldsymbol{\sigma}=\left(m_{2} / m_{1}\right) \mathbf{S}_{\mathbf{1}}+$ $\left(m_{1} / m_{2}\right) \mathbf{S}_{\mathbf{2}}$. The magnitude and direction of the magnetic dipole moments $\mathbf{d}_{\mathbf{i}}$ are denoted as $d_{i}$ and $\hat{\mathbf{d}}_{\mathbf{i}}$. They subtend the angle $\lambda=\cos ^{-1}\left(\hat{\mathbf{d}}_{\mathbf{1}} \cdot \hat{\mathbf{d}}_{\mathbf{2}}\right)$ with each other. In a coordinate systems $\mathcal{K}$ with the axes $(\hat{\mathbf{c}}, \hat{\mathbf{L}} \times \hat{\mathbf{c}}, \hat{\mathbf{L}})$, where $\hat{\mathbf{c}}$ is the unit vector in the $\mathbf{J} \times \mathbf{L}$ direction, the polar angles $\kappa_{i}$ and $\psi_{i}$ of the spins are defined as $\hat{\mathbf{S}}_{\mathbf{i}}=$ $\left(\sin \kappa_{i} \cos \psi_{i}, \sin \kappa_{i} \sin \psi_{i}, \cos \kappa_{i}\right)$ (see [19]). In the coordinate system $\mathcal{K}^{i}$ with the axes $\left(\hat{\mathbf{b}}_{\mathbf{i}}, \hat{\mathbf{S}}_{\mathbf{i}} \times \hat{\mathbf{b}}_{\mathbf{i}}, \hat{\mathbf{S}}_{\mathbf{i}}\right)$, where $\hat{\mathbf{b}}_{\mathbf{i}}$ are the unit vectors in the $\mathbf{S}_{\mathbf{i}} \times \mathbf{L}$ directions, respectively, the polar angles $\alpha_{i}$ and $\beta_{i}$ of the the magnetic dipole moments $\mathbf{d}_{\mathbf{i}}$ are $\hat{\mathbf{d}}_{\mathbf{i}}=\left(\sin \alpha_{i} \cos \beta_{i}, \sin \alpha_{i} \sin \beta_{i}, \cos \alpha_{i}\right)$ (see [27]). The quadrupolar parameters (see [26]) are defined as $p_{i}=Q_{i} / m_{i} m^{2}$, where $Q_{i}$ is the quadrupole-moment scalar [33] of the $i^{\text {th }}$ axially symmetric binary component with symmetry axis $\hat{\mathbf{S}}_{\mathbf{i}}$. The reduced mass is $\mu=m_{1} m_{2} / m$ and $\eta=\mu / m$.
From (3) a radial equation can be derived

$$
\dot{r}^{2}=\frac{2 E}{\mu}+\frac{2 G m}{r}-\frac{\bar{L}^{2}}{\mu^{2} r^{2}}+\sum_{i=0}^{3} \frac{\delta A_{i}}{r^{i}}-\frac{2 \bar{L} \delta L}{\mu^{2} r^{2}}-\frac{2 \delta E}{\mu} .
$$

Here $\bar{L}=(1 / 2 \pi) \int_{0}^{2 \pi} L(\chi) d \chi$ is the angular average of the magnitude of orbital angular momentum $L(\chi), \chi$ being the true anomaly parameter. The explicit values of $\bar{L}$ in the case of spin-spin, quadrupole-monopole, and magnetic dipole-dipole interactions were computed in [25-27]. $\bar{A}$ is the magnitude of the Laplace-Runge-Lenz vector characterizing a Keplerian motion with $E$ and $\bar{L}$. The coefficients $\delta A_{i}$ in Eq. (4) are constant PN perturbations given in the Table I, which can be read from [31]. The SO, SS, QM and DD contributions to $\delta L$ and $\delta E$ are enlisted in the Tables II and III. The shorthand notations $\alpha_{\mathrm{DD}}$ and $\beta_{\mathrm{DD}}(2 \chi)$ are defined in Eq. (6).

TABLE I. Various post-Newtonian constants in $\delta A_{i}$.

\begin{tabular}{lc}
\hline \hline$\delta A_{0}$ & $3(3 \eta-1) \frac{E^{2}}{c^{2} \mu^{2}}$ \\
$\delta A_{1}$ & $2(7 \eta-6) \frac{E G m}{c^{2} \mu}$ \\
$\delta A_{2}$ & $-2(3 \eta-1) \frac{E L^{2}}{c^{2} \mu^{3}}+(5 \eta-10) \frac{G^{2} m^{2}}{c^{2}}$ \\
$\delta A_{3}$ & $(-3 \eta+8) \frac{G^{2} m^{2} \bar{L}^{2}}{c^{2} \mu^{2}}$ \\
\hline \hline
\end{tabular}


TABLE II. Various contributions to $\delta L$.

\begin{tabular}{lc}
\hline \hline SO & $\frac{G \mu \bar{L}}{2 c^{2} r} \sum_{i=1, j \neq i}^{2} \frac{4 m_{i}+3 m_{j}}{m_{i}} S_{i} \cos \kappa_{i}$ \\
SS & $-\frac{G \mu^{2}}{2 c^{2} \bar{L}^{3}} S_{1} S_{2} \sin \kappa_{1} \sin \kappa_{2}\left\{2 \bar{A} \cos \left[\chi+2\left(\psi_{0}-\bar{\psi}\right)\right]\right.$ \\
& $\left.+(3 G m \mu+2 \bar{A} \cos \chi) \cos 2\left(\chi+\psi_{0}-\bar{\psi}\right)\right\}$ \\
QM & $\frac{G \mu^{3} m^{3}}{4 \bar{L}^{3}} \sum_{i=1}^{2} p_{i} \sin ^{2} \kappa_{i}\left\{2 \bar{A} \cos \left[\chi+2\left(\psi_{0}-\psi_{i}\right)\right]\right.$ \\
& $\left.+(3 G m \mu+2 \bar{A} \cos \chi) \cos 2\left(\chi+\psi_{0}-\psi_{i}\right)\right\}$ \\
DD & $\frac{\mu^{2} d_{1} d_{2}}{2 \bar{L}^{3}}\left[(3 G m \mu+4 \bar{A} \cos \chi) \beta_{\mathrm{DD}}(2 \chi)-\bar{A} \sin \chi \frac{d \beta_{D D}(2 \chi)}{d \chi}\right]$ \\
\hline
\end{tabular}

In [36] a generic scheme was introduced for parametrizing such perturbed Keplerian motions. The advantage of the generalized eccentric anomaly parametrization and generalized true anomaly parametrization is that a simple technique based on the residue theorem can be applied for computing secular effects [36]. The scheme was applied individually for each of the SO, SS, QM, DD perturbations in [19,25-27], respectively. It is straightforward to derive the PN contribution to these parametrizations. We give here in a concise form both parametrizations, with the inclusion of the PN contribution as well. Both are defined in terms of the turning points $r_{\min }$ and $r_{\max }$ of the radial motion, given by $\dot{r}=0$ :

$$
\begin{aligned}
& r_{\operatorname{mix}}=\frac{G m \mu \pm \bar{A}}{-2 E}+\delta r_{\min }^{\mathrm{PN}}+\delta r_{\min }^{\mathrm{SO}}+\delta r_{\min }^{\mathrm{SO}}+\delta r_{\text {max }}^{\mathrm{SS}} \\
& +\delta r_{\substack{\max \\
\text { min }}}^{\mathrm{DD}} \\
& \delta r_{\substack{\text { max } \\
\text { min }}}^{\mathrm{PN}}=(\eta-7) \frac{G m}{4 c^{2}} \pm(\eta+9) \frac{G^{2} m^{2} \mu}{8 \bar{A} c^{2}} \\
& \mp(3 \eta-1) \frac{\bar{A}}{8 \mu c^{2}} \text {, } \\
& \delta r_{\substack{\text { mix } \\
\text { min }}}^{\mathrm{SO}}=-\frac{G \mu}{2 c^{2} \bar{L} \bar{A}}(\bar{A} \mp G m \mu) \sum_{i=1, j \neq i}^{2} \frac{4 m_{i}+3 m_{j}}{m_{i}} S_{i} \cos \kappa_{i}, \\
& \delta r_{\min }^{\mathrm{SS}}=-\frac{G \mu S_{1} S_{2}}{2 c^{2} \bar{L}^{2} \bar{A}}\left[(\bar{A} \mp G m \mu) \alpha_{\mathrm{SS}}+\bar{A} \beta_{\mathrm{SS}}\right], \\
& \delta r_{\substack{\max \\
\text { min }}}^{\mathrm{QM}}=\frac{G \mu^{2} m^{3}}{4 \bar{L}^{2} \bar{A}} \sum_{i=1}^{2} p_{i}\left[(\bar{A} \mp G m \mu) \alpha_{\mathrm{QM}}^{i}+\bar{A} \beta_{\mathrm{QM}}^{i}\right] \text {, } \\
& \delta r_{\substack{\text { max } \\
\text { min }}}^{\mathrm{DD}}=\frac{\mu d_{1} d_{2}}{2 \bar{L}^{2} \bar{A}}\left\{(\bar{A} \mp G m \mu) \alpha_{\mathrm{DD}}+\bar{A} \beta_{\mathrm{DD}}\right\} .
\end{aligned}
$$

We have introduced the shorthand notations

TABLE III. Various contributions to $\delta E$.

\begin{tabular}{lc}
\hline \hline SO & No contribution \\
SS & $-\frac{G S_{1} S_{2}}{2 c^{2} r^{3}}\left\{3 \cos \kappa_{1} \cos \kappa_{2}-\cos \gamma\right.$ \\
& $\left.-3 \sin \kappa_{1} \sin \kappa_{2} \cos 2\left(\chi+\psi_{0}-\bar{\psi}\right)\right\}$ \\
QM & $\frac{G \mu m^{3}}{2 r^{3}} \sum_{i=1}^{2} p_{i}\left[1-3 \sin ^{2} \kappa_{i} \cos ^{2}\left[\chi+2\left(\psi_{0}-\psi_{i}\right)\right]\right]$ \\
DD & $\frac{d_{1} d_{2}}{2 r^{3}}\left[\alpha_{\text {DD }}-3 \beta_{\mathrm{DD}}(2 \chi)\right]$ \\
\hline \hline
\end{tabular}

$$
\begin{aligned}
\alpha_{\mathrm{SS}}= & 3 \cos \kappa_{1} \cos \kappa_{2}-\cos \gamma, \\
\beta_{\mathrm{SS}}= & \sin \kappa_{1} \sin \kappa_{2} \cos 2\left(\psi_{0}-\bar{\psi}\right), \\
\alpha_{\mathrm{QM}}^{i}= & 2-3 \sin ^{2} \kappa_{i}, \quad \beta_{\mathrm{QM}}^{i}=\sin ^{2} \kappa_{i} \cos 2\left(\psi_{0}-\psi_{i}\right), \\
\alpha_{\mathrm{DD}}= & 2 \cos \lambda+3\left(\rho_{1} \sigma_{2}-\rho_{2} \sigma_{1}\right) \sin \Delta \psi \\
& -3\left(\rho_{1} \rho_{2}+\sigma_{1} \sigma_{2}\right) \cos \Delta \psi, \\
\beta_{\mathrm{DD}}(k \chi)= & \left(\sigma_{1} \sigma_{2}-\rho_{1} \rho_{2}\right) \cos \left[k \chi+2\left(\psi_{0}-\bar{\psi}\right)\right] \\
& -\left(\rho_{1} \sigma_{2}+\rho_{2} \sigma_{1}\right) \sin \left[k \chi+2\left(\psi_{0}-\bar{\psi}\right)\right], \\
\beta_{\mathrm{DD}}= & \beta_{\mathrm{DD}}(0),
\end{aligned}
$$

where

$$
\begin{aligned}
\rho_{i} & =\sin \alpha_{i} \cos \beta_{i}, \\
\sigma_{i} & =\cos \alpha_{i} \sin \kappa_{i}+\sin \alpha_{i} \sin \beta_{i} \cos \kappa_{i} .
\end{aligned}
$$

The generalized eccentric anomaly parametrization $r(\xi)$ is then defined as

$$
r(\xi)=a_{r}\left(1-e_{r} \cos \xi\right) .
$$

The eccentric anomaly $\xi$ reduces to the eccentric anomaly parameter $u$ of [31] for $1 \mathrm{PN}$ perturbations. In Eq. (8) the semimajor axis $a_{r}$ and the radial eccentricity $e_{r}$ was introduced as

$$
\begin{aligned}
& a_{r}=\frac{r_{\max }+r_{\text {min }}}{2}, \\
& e_{r}=\frac{r_{\text {max }}-r_{\text {min }}}{r_{\text {max }}+r_{\text {min }}} .
\end{aligned}
$$

The generalized true anomaly parametrization is defined as

$$
\frac{2}{r(\chi)}=\left(\frac{1}{r_{\min }}+\frac{1}{r_{\max }}\right)+\left(\frac{1}{r_{\min }}-\frac{1}{r_{\max }}\right) \cos \chi .
$$

These two parametrizations of the radial motion are interrelated by the Keplerian relations

$$
\begin{aligned}
\tan \frac{\xi}{2} & =\sqrt{\frac{1-e_{r}}{1+e_{r}}} \tan \frac{\chi}{2}, \\
\sin \xi & =\frac{\sqrt{1-e_{r}^{2}} \sin \chi}{1+e_{r} \cos \chi},
\end{aligned}
$$

with $e_{r}$ in place of the Keplerian eccentricity. Note that in the Damour-Deruelle formalism [31], a different generalized true anomaly parameter $v$ is introduced. To 1PN accuracy $v$ is related to $\chi$ as

$$
\tan \frac{\chi}{2}=\left[1-\frac{G m \mu}{4 c^{2} \bar{L}^{2} \bar{A}}\left(G^{2} m \mu^{3}+\frac{12 E \bar{L}^{2}}{\mu}\right)\right] \tan \frac{v}{2} .
$$

When using the generalized true anomaly parameter $v$, the equation $\xi(v)$ replacing Eq. (12) will contain the angular eccentricity $e_{\theta}$ rather than $e_{r}$. 


\section{GENERALIZED KEPLER EQUATION}

The first three terms on the right hand side in the radial Eq. (4) sum up to

$$
\begin{aligned}
\frac{2 E}{\mu}+\frac{2 G m}{r}-\frac{\bar{L}^{2}}{\mu^{2} r^{2}}= & \left(\frac{\bar{A}}{\bar{L}^{2}}+\frac{2 \delta Q}{\mu}\right) \bar{A} \sin ^{2} \chi \\
& -\frac{2 \bar{A}}{\mu}(\delta Q+\delta P \cos \chi),
\end{aligned}
$$

where $\delta P$ and $\delta Q$ are perturbation terms depending on the physical parameters of the binary and they are given in the Tables IV and V. The parametrization (11) has the advantageous property

$$
\frac{d r}{d \chi}=\frac{1}{2}\left(\frac{1}{r_{\min }}-\frac{1}{r_{\max }}\right) r^{2} \sin \chi .
$$

Employing Eqs. (11) and (16) into the radial Eq. (4), then taking the square root and forming the reciprocal, after a series expansion to first order in the perturbations we find

$$
\begin{aligned}
\frac{d t}{d \chi}= & \frac{\mu r^{2}}{\bar{L}}\left\{1+\frac{\bar{L}^{2}}{2 \mu^{2} r^{2} \bar{A}^{2} \sin ^{2} \chi}\left[r^{2} \mu \bar{A}(\delta Q+\delta P \cos \chi)\right.\right. \\
& \left.\left.+2\left(\bar{L} \delta L+r^{2} \mu \delta E\right)+\sum_{i=0}^{3} \delta A_{i} r^{2-i}\right]\right\} .
\end{aligned}
$$

The integration of this equation is the main purpose of our paper.

Apparently Eq. (17) becomes singular at $\chi=k \pi, k \in$ $Z$, because of the $\sin ^{2} \chi$ term in the denominator. Such singularities could be just apparent, as was shown for the SO contribution in [36]. We have verified that after forming the common denominator in the bracket, and inserting the detailed expressions of $\delta P, \delta Q, \delta L$, and $\delta E$, given in Tables II, III, IV, and V, the numerator becomes proportional to $\sin ^{2} \chi$. Therefore the singularities are just apparent rather than real for all type of contributions considered here. Thus we obtain

$$
\begin{aligned}
\frac{d t}{d \chi} & =\frac{\mu r^{2}}{\bar{L}}+\left(\frac{d t}{d \chi}\right)_{\mathrm{PN}}+\left(\frac{d t}{d \chi}\right)_{\mathrm{SO}}+\left(\frac{d t}{d \chi}\right)_{\mathrm{SS}}+\left(\frac{d t}{d \chi}\right)_{\mathrm{DD}}+\left(\frac{d t}{d \chi}\right)_{\mathrm{QM}}, \\
\left(\frac{d t}{d \chi}\right)_{\mathrm{PN}} & =\frac{\mu r^{2}}{2 c^{2} \bar{L}^{3}}\left\{(\eta-13) G^{2} m^{2} \mu^{2}+(3 \eta-1) \bar{A}^{2}+(3 \eta-8) G m \mu \bar{A} \cos \chi\right\}, \\
\left(\frac{d t}{d \chi}\right)_{\mathrm{SO}} & =-\frac{G \mu^{2} r^{2}}{2 c^{2} \bar{L}^{3}}(3 G m \mu+\bar{A} \cos \chi) \sum_{i=1, j \neq i}^{2} \frac{4 m_{i}+3 m_{j}}{m_{i}} S_{i} \cos \kappa_{i}, \\
\left(\frac{d t}{d \chi}\right)_{\mathrm{SS}} & =\frac{G \mu^{3} S_{1} S_{2} r^{2}}{2 c^{2} \bar{L}^{5}}\left\{\left[G m \mu\left(3 \alpha_{\mathrm{SS}}+2 \beta_{\mathrm{SS}}\right)+\bar{A}\left(\alpha_{\mathrm{SS}}+\beta_{\mathrm{SS}}\right) \cos \chi\right]-\frac{2 \bar{L}^{4}}{\mu^{2} r^{2} \bar{A}} \sin \kappa_{1} \sin \kappa_{2} \cos \left[\chi+2\left(\psi_{0}-\bar{\psi}\right)\right]\right\}, \\
\left(\frac{d t}{d \chi}\right)_{\mathrm{QM}} & =-\frac{G m^{3} \mu^{4} r^{2}}{4 \bar{L}^{5}} \sum_{i=1}^{2} p_{i}\left\{\left[G m \mu\left(3 \alpha_{\mathrm{QM}}^{i}+2 \beta_{\mathrm{QM}}^{i}\right)+\bar{A}\left(\alpha_{\mathrm{QM}}^{i}+\beta_{\mathrm{QM}}^{i}\right) \cos \chi\right]-\frac{2 \bar{L}^{4}}{\mu^{2} r^{2} \bar{A}} \sin ^{2} \kappa_{i} \cos \left[\chi+2\left(\psi_{0}-\psi_{i}\right)\right]\right\}, \\
\left(\frac{d t}{d \chi}\right)_{\mathrm{DD}} & =-\frac{\mu^{3} d_{1} d_{2} r^{2}}{2 \bar{L}^{5}}\left\{\left[G m \mu\left(3 \alpha_{\mathrm{DD}}+2 \beta_{\mathrm{DD}}\right)+\bar{A}\left(\alpha_{\mathrm{DD}}+\beta_{\mathrm{DD}}\right) \cos \chi\right]+\frac{2 \bar{L}^{4}}{\mu^{2} r^{2} \bar{A}} \cos \left[\chi+2\left(\psi_{0}-\bar{\psi}\right)\right]\right\} .
\end{aligned}
$$

\begin{tabular}{|c|c|}
\hline PN & $-\frac{\mu}{8 c^{2} \bar{L}^{4} \bar{A}}\left[2 G^{2} m^{2} \mu^{2} \bar{A}^{2}(3 \eta-19)-G^{4} m^{4} \mu^{4}(\eta+9)+\bar{A}^{4}(3 \eta-1)\right]$ \\
\hline SO & $-\frac{G^{2} m \mu^{4}}{2 c^{2} \bar{T}^{5 \bar{A}}} \sum_{i=1, j \neq i}^{2} \frac{4 m_{i}+3 m_{j}}{m_{i}} S_{i} \cos \kappa_{i}\left(3 \bar{A}^{2}+G^{2} m^{2} \mu^{2}\right)$ \\
\hline SS & $\frac{G^{2} m \mu^{4} S_{1} S_{2}}{2 c^{2} \bar{T}^{6} \overline{\bar{A}}}\left[\alpha_{\mathrm{SS}}\left(3 \bar{A}^{2}+G^{2} m^{2} \mu^{2}\right)+2 \beta_{\mathrm{QM}} \bar{A}^{2}\right]$ \\
\hline $\mathrm{QM}$ & $-\frac{G^{2} m^{4} \mu^{5}}{4 \bar{T}^{6} \bar{A}} \sum_{i=1}^{2} p_{i}\left[\alpha_{\mathrm{OM}}^{i}\left(3 \bar{A}^{2}+G^{2} m^{2} \mu^{2}\right)+2 \beta_{\mathrm{SS}}^{i} \bar{A}^{2}\right]$ \\
\hline DD & $-\frac{G m \mu^{4} d_{1} d_{2}}{2 \bar{L}^{6} \bar{A}}\left[\alpha_{\mathrm{DD}}\left(3 \bar{A}^{2}+G^{2} m^{2} \mu^{2}\right)+2 \beta_{\mathrm{DD}} \bar{A}^{2}\right]$ \\
\hline
\end{tabular}

TABLE IV. Various contributions to $\delta P$.

\begin{tabular}{lc}
\hline \hline PN & $-\frac{G m \mu^{2}}{c^{2} \bar{L}^{4}}\left[\bar{A}^{2}(\eta-2)-4 G^{2} m^{2} \mu^{2}\right]$ \\
SO & $-\frac{G \mu^{3}}{2 c^{2} \bar{L}^{5}} \sum_{i=1, j \neq i}^{2} \frac{4 m_{i}+3 m_{j}}{m_{i}} S_{i} \cos \kappa_{i}\left(\bar{A}^{2}+3 G^{2} m^{2} \mu^{2}\right)$ \\
SS & $\frac{G \mu^{3} S_{1} S_{2}}{2 c^{2}}\left[\alpha_{\mathrm{SS}}\left(\bar{A}^{2}+3 G^{2} m^{2} \mu^{2}\right)+\beta_{\mathrm{SS}}\left(\bar{A}^{2}+G^{2} m^{2} \mu^{2}\right)\right]$ \\
QM & $-\frac{G m^{3} \mu^{4} \mu^{6}}{4 \bar{L}^{6}} \sum_{i=1}^{2} p_{i}\left[\alpha_{\mathrm{QM}}^{i}\left(\bar{A}^{2}+3 G^{2} m^{2} \mu^{2}\right)+\beta_{\mathrm{QM}}^{i}\left(\bar{A}^{2}+G^{2} m^{2} \mu^{2}\right)\right]$ \\
DD & $-\frac{\mu^{3} d_{1} d_{2}}{2 \bar{L}^{6}}\left[\alpha_{\mathrm{DD}}\left(\bar{A}^{2}+3 G^{2} m^{2} \mu^{2}\right)+\beta_{\mathrm{DD}}\left(\bar{A}^{2}+G^{2} m^{2} \mu^{2}\right)\right]$ \\
\hline \hline
\end{tabular}

TABLE V. Various contributions to $\delta Q$. 
These expressions are regular for any $\chi$.

By integrating Eq. (18) and employing the relation (12) between the two parametrizations we obtain the generalized Kepler equation (1). This is the main result of the paper.

\section{THE ORBITAL PARAMETERS}

In this section we enlist the detailed expressions of the orbital parameters appearing either in the eccentric anomaly parametrization (8) or in the generalized Kepler equation (1).

The semimajor axis is

$$
\begin{aligned}
& a_{r}=\frac{G m \mu}{-2 E}+a_{r}^{\mathrm{PN}}+a_{r}^{\mathrm{SO}}+a_{r}^{\mathrm{SS}}+a_{r}^{\mathrm{QM}}+a_{r}^{\mathrm{DD}}, \\
& a_{r}^{\mathrm{PN}}=\frac{G m}{4 c^{2}}(\eta-7), \\
& a_{r}^{\mathrm{SO}}=\frac{G \mu}{2 c^{2} \bar{L}} \sum_{i=1, j \neq i}^{2} \frac{4 m_{i}+3 m_{j}}{m_{i}} S_{i} \cos \kappa_{i}, \\
& a_{r}^{\mathrm{SS}}=-\frac{G \mu S_{1} S_{2}}{2 c^{2} \bar{L}^{2}}\left(\alpha_{\mathrm{SS}}+\beta_{\mathrm{SS}}\right), \\
& a_{r}^{\mathrm{QM}}=\frac{G m^{3} \mu^{2}}{4 \bar{L}^{2}} \sum_{i=1}^{2} p_{i}\left(\alpha_{\mathrm{QM}}^{i}+\beta_{\mathrm{QM}}^{i}\right), \\
& a_{r}^{\mathrm{DD}}=\frac{\mu d_{1} d_{2}}{2 \bar{L}^{2}}\left(\alpha_{\mathrm{DD}}+\beta_{\mathrm{DD}}\right) .
\end{aligned}
$$

The radial eccentricity is

$$
\begin{aligned}
& e_{r}=\frac{\bar{A}}{G m \mu}+e_{r}^{\mathrm{PN}}+e_{r}^{\mathrm{SO}}+e_{r}^{\mathrm{SS}}+e_{r}^{\mathrm{QM}}+e_{r}^{\mathrm{DD}}, \\
& e_{r}^{\mathrm{PN}}=\frac{E}{4 c^{2} G m \mu^{2} \bar{A}}\left[(5 \eta-15) \bar{A}^{2}-(\eta+9) G^{2} m^{2} \mu^{2}\right], \\
& e_{r}^{\mathrm{SO}}=\frac{E\left(G^{2} m^{2} \mu^{2}+\bar{A}^{2}\right)}{c^{2} G m^{2} \mu \bar{L} \bar{A}} \sum_{i=1, j \neq i}^{2} \frac{4 m_{i}+3 m_{j}}{m_{i}} S_{i} \cos \kappa_{i}, \\
& e_{r}^{\mathrm{SS}}=-\frac{E S_{1} S_{2}}{c^{2} G m^{2} \mu \bar{L}^{2} \bar{A}}\left[\left(G^{2} m^{2} \mu^{2}+\bar{A}^{2}\right) \alpha_{\mathrm{SS}}+\bar{A}^{2} \beta_{\mathrm{SS}}\right], \\
& e_{r}^{\mathrm{QM}}=\frac{E m}{2 G \bar{L}^{2} \bar{A}} \sum_{i=1}^{2} p_{i}\left[\left(G^{2} m^{2} \mu^{2}+\bar{A}^{2}\right) \alpha_{\mathrm{QM}}^{i}+\bar{A}^{2} \beta_{\mathrm{QM}}^{i}\right], \\
& e_{r}^{\mathrm{DD}}=\frac{E d_{1} d_{2}}{G m \mu \bar{L}^{2} \bar{A}}\left[\left(G^{2} m^{2} \mu^{2}+\bar{A}^{2}\right) \alpha_{\mathrm{DD}}+\bar{A}^{2} \beta_{\mathrm{DD}}\right] .
\end{aligned}
$$

The mean motion is

$$
n=\frac{2 \pi}{T}=\frac{1}{G m}\left(\frac{-2 E}{\mu}\right)^{3 / 2}\left[1-(\eta-15) \frac{E}{4 c^{2} \mu}\right] .
$$

The time eccentricity is

$$
\begin{aligned}
e_{t} & =\frac{\bar{A}}{G m \mu}+e_{t}^{\mathrm{PN}}+e_{t}^{\mathrm{SO}}+e_{t}^{\mathrm{SS}}+e_{t}^{\mathrm{QM}}+e_{t}^{\mathrm{DD}} \\
e_{t}^{\mathrm{PN}} & =-\frac{E}{4 c^{2} G m \mu^{2} \bar{A}}\left[(7 \eta-17) \bar{A}^{2}+(\eta+9) G^{2} m^{2} \mu^{2}\right] \\
e_{t}^{\mathrm{SO}} & =\frac{E G \mu}{c^{2} \bar{L} \bar{A}} \sum_{i=1, j \neq i}^{2} \frac{4 m_{i}+3 m_{j}}{m_{i}} S_{i} \cos \kappa_{i} \\
e_{t}^{\mathrm{SS}} & =-\frac{E S_{1} S_{2} G \mu \alpha_{\mathrm{SS}}}{c^{2} \bar{L}^{2} \bar{A}} \\
e_{t}^{\mathrm{QM}} & =\frac{E G m^{3} \mu^{2}}{2 \bar{L}^{2} \bar{A}} \sum_{i=1}^{2} p_{i} \alpha_{\mathrm{QM}}^{i}, \\
e_{t}^{\mathrm{DD}} & =\frac{E \mu d_{1} d_{2}}{\bar{L}^{2} \bar{A}} \alpha_{\mathrm{DD}} .
\end{aligned}
$$

In what follows, we enlist the parameters $f_{t}$ and $f_{t}^{i}$, the analogues of which also appear in the extension to 2PN [37] of the Damour-Deruelle parametrization, however have no PN counterpart. The parameter $f_{t}$ receives only SS and DD type contributions

$$
\begin{aligned}
f_{t} & =f_{t}^{\mathrm{SS}}+f_{t}^{\mathrm{DD}}, \\
f_{t}^{\mathrm{SS}} & =-\left(\frac{-2 E}{\mu}\right)^{3 / 2} \frac{\mu S_{1} S_{2}}{c^{2} m \bar{A} \bar{L}} \sin \kappa_{1} \sin \kappa_{2}, \\
f_{t}^{\mathrm{DD}} & =\left(\frac{-2 E}{\mu}\right)^{3 / 2} \frac{\mu d_{1} d_{2}}{G m \bar{A} \bar{L}} .
\end{aligned}
$$

Finally the parameters $f_{t}^{i}$, originating from the QM interaction, are given as

$$
f_{t}^{i}=\left(\frac{-2 E}{\mu}\right)^{3 / 2} \frac{m^{2} \mu^{2}}{2 \bar{A} \bar{L}} p_{i} \sin ^{2} \kappa_{i}
$$

\section{CONCLUDING REMARKS}

The generalized Kepler equation (1) with the orbital parameters (19)-(24), together with any of the parametrizations (8) or (11) and their relation (12) represent the complete solution of the radial motion of the compact binary on eccentric orbit, to linear order in the perturbations. All perturbations arising from relativistic corrections and from the presence of spins, mass quadrupole and magnetic dipole moments are included here to linear order (PN, SO, SS, QM, DD contributions).

The generalized Kepler equation contains two parameters, the generalized eccentric anomaly $\xi$ (this is defined similarly as the parameter $u$ in $[31,37]$ and the generalized true anomaly $\chi$ (different from the parameter $v$ of $[31,37]$, their relation being given to 1PN accuracy by Eq. (14)).

The generalization of the relation (14) to $2 \mathrm{PN}$ accuracy would imply to give the $\chi$ parametrization (11) to $2 \mathrm{PN}$, however by the method described in [36] $r(\chi)$ can be defined only to linear order in the perturbations. 
Nevertheless, the linear contributions to the Kepler equation included in the present paper (containing the parameters $\xi$ and $\chi$ ), and the 2PN contributions [37] (containing $u \equiv \xi$ and $v$ ) can be simply summed up, at the price of having all three parameters $(\xi \equiv u, \chi$, and $v)$ present in the formalism. Notably at $2 \mathrm{PN}$ orders a new parameter, $g_{t}$ is also present [38]. We note here that the 3PN contribution to the Kepler equation is also known [15], however the contribution of the first PN correction of the SO interaction, arising at $2.5 \mathrm{PN}$ is not. Therefore we conclude that for the moment, the radial motion is solved only to $2 \mathrm{PN}$ orders accuracy.

In contrast with the $\mathrm{PN}$ and 2PN Kepler equations, our Eq. (1) contains the additional angles $\psi_{0}$ and $\psi_{i}$. During one radial period these can be considered constants [19]. On the long run however, all these angles slowly vary as the orbit and the spins undergo precessional motions. In order to describe the slow evolution of these angles, the study of the angular part of the motion (as opposed to the radial one) is necessary, with the inclusion of all perturbations, to linear order. This is available for the PN perturbation
[31] and SO perturbation [15], however the latter holds only for special cases (equal masses or a single spin). A systematic investigation of the angular part of the perturbed Keplerian motion is under way [39].

We remark that the circular orbit limit of our formulae should not be taken as $\bar{A} \rightarrow 0$. This is because the standard interpretation of the Laplace-Runge-Lenz vector holds only in the Newtonian limit. In the circular orbit limit all corrections considered here add nonvanishing contributions to $\bar{A}$.

Our generic Kepler equation (1) and the orbital elements (19)-(24) correctly reproduce the PN contributions [31] and the SO contributions [15]. However all of the other contributions are new.

\section{ACKNOWLEDGMENTS}

This work was supported by OTKA Grants No. T046939 and TS044665. L. Á. G. was further supported by the János Bolyai Grant from the Hungarian Academy of Sciences.
[1] A. Abramovici et al., Science 256, 325 (1992).

[2] K. Kuroda et al., in Proceedings of International Conference on Gravitational Waves: Sources and Detectors, edited by I. Ciufolini and F. Fidecaro (World Scientific, Singapore, 1997), p. 100.

[3] C. Bradaschia et al., Nucl. Instrum. Methods A 289, 518 (1990).

[4] J. Hough, in Proceedings of the Sixth Marcell Grossmann Meeting, edited by H. Sato and T. Nakamura (World Scientific, Singapore, 1992) p. 192.

[5] B. Abbott et al. (LIGO Scientific Collaboration), gr-qc/ 0505042 .

[6] B. Abbott et al., (LIGO Scientific Collaboration), gr-qc/ 0509129.

[7] P. R. Brady, J. D.E. Creighton, and K. S. Thorne, Phys. Rev. D 58, 061501 (1998).

[8] A. Buonanno, Y. Chen, and M. Vallisneri, Phys. Rev. D 67, 024016 (2003).

[9] L. Blanchet, B. R. Iyer, B. Joguet, Phys. Rev. D 65, 064005 (2002); 71, 129903E (2005).

[10] B. M. Barker and R. F. O'Connell, Phys. Rev. D 2, 1428 (1970).

[11] R. F. O'Connell, Phys. Rev. Lett. 93, 081103 (2004).

[12] N. Burgay et al., Nature (London) 426, 531 (2003).

[13] A. G. Lyne et al., Science 303, 1153 (2004).

[14] A. Apostolatos, C. Cutler, G. J. Sussman, and K. S. Thorne, Phys. Rev. D 49, 6274 (1994).

[15] C. Köenigsdörffer and A. Gopakumar, Phys. Rev. D 71, 024039 (2005). For the conversion to our notations we remark that $2 \mathbf{S}_{e f f} \cdot \mathbf{L}=4 \mathbf{L} \cdot \mathbf{S}+3 \mathbf{L} \cdot \sigma$.

[16] C. Köenigsdörffer and A. Gopakumar, gr-qc/0509012.

[17] P. Grandclement, M. Ihm, V. Kalogera, and K. Belczynski,
Phys. Rev. D 69, 102002, (2004).

[18] R. Rieth and G. Schafer, Classical Quantum Gravity 14, 2357, (1997).

[19] L. Á. Gergely, Z. I. Perjés, and M. Vasúth, Phys. Rev. D 57, 876 (1998); L. Á. Gergely, Z. I. Perjés, and M. Vasúth, Phys. Rev. D 57, 3423 (1998); L. Á. Gergely, Z. I. Perjés, and M. Vasúth, Phys. Rev. D 58, 124001 (1998).

[20] A. Buonanno and T. Damour, Phys. Rev. D 59, 084006 (1999).

[21] A. Buonanno and T. Damour, Phys. Rev. D 62, 064015 (2000).

[22] A. Buonanno, Y. Chen, and T. Damour, gr-qc/0508067.

[23] A. Buonanno, Y. Chen, and M. Vallisneri, Phys. Rev. D 67, 104025 (2003).

[24] A. Buonanno, Y. Chen, Y. Pan, H. Tagoshi, and M. Vallisneri, Phys. Rev. D 72, 084027 (2005).

[25] L. Á. Gergely, Phys. Rev. D 61, 024035 (2000); L. Á. Gergely, Phys. Rev. D 62, 024007 (2000).

[26] L. Á. Gergely and Z. Keresztes, Phys. Rev. D 67, 024020 (2003).

[27] M. Vasúth, Z. Keresztes, A. Mihály, and L. Á. Gergely, Phys. Rev. D 68, 124006 (2003).

[28] B. Mikóczi, M. Vasúth, and L. Á. Gergely, Phys. Rev. D 71, 124043 (2005).

[29] M. H. L. Pryce, Proc. R. Soc. A 195, 62 (1948).

[30] T. D. Newton and E. P. Wigner, Rev. Mod. Phys. 21, 400 (1949).

[31] T. Damour and N. Deruelle, Ann. Inst. Henri Poincaré A 43,107 (1985).

[32] L. Kidder, C. Will, and A. Wiseman, Phys. Rev. D 47, R4183 (1993).

[33] E. Poisson, Phys. Rev. D 57, 5287 (1998). 
[34] K. Ioka and T. Taniguchi, Astrophys. J. 537, 327 (2000).

[35] L. Kidder, Phys. Rev. D 52, 821 (1995).

[36] L. Á. Gergely, Z. I. Perjés, and M. Vasúth, Astrophys. J. Suppl. Ser. 126, 79 (2000).

[37] T. Damour and G. Schäfer, Nouvo Cimento B 101, 127
(1988).

[38] G. Schäfer and N. Wex, Phys. Lett. A 174, 196 (1993); 177, 461E (1993).

[39] Z. Keresztes, B. Mikóczi, and L.Á. Gergely (to be published). 